\title{
Development and Characterization of a Small Spacecraft Electro-Optic Scanner for Free-Space Laser Communications
}

\author{
Scott Davis ${ }^{1}$ \\ Vescent Photonics, Inc., 14998 West 6th Ave, Unit 700, Golden, CO, 80401 \\ Michael Lichter ${ }^{2}$ and Daniel Raible ${ }^{3}$ \\ NASA John H. Glenn Research Center at Lewis Field, 21000 Brookpark Road, \\ Cleveland, $\mathrm{OH}, 44135$
}

\begin{abstract}
Emergent data-intensive missions coupled with dramatic reductions in spacecraft size plus an increasing number of space-based missions necessitates new high performance, compact and low cost communications technology. Free space optical communications offer advantages including orders of magnitude increase for data rate performance, increased security, immunity to jamming and lack of frequency allocation requirements when compared with conventional radio frequency (RF) means. The spatial coherence and low divergence associated with the optical frequencies of laser communications lends themselves to superior performance, but this increased directionality also creates one of the primary technical challenges in establishing a laser communications link by repeatedly and reliably pointing the beam onto the receive aperture. Several solutions have emerged from wide angle (slow) mechanical articulation systems, fine (fast) steering mirrors and rotating prisms, inertial compensation gyros and vibration isolation \& cancellation systems, but each requires moving components and imparts a measured amount of burden on the host platform. The complexity, cost and size of current mechanically scanned solutions limits their platform applicability, and restricts the feasibility of deploying optical communications payloads on very compact
\end{abstract}

1

Vice President of Technology \& Co-Founder, Vescent Photonics, davis@ vescent.com, Not an AIAA Member.

2

Electronics Engineer, Avionics Branch (LEA), NASA GRC, michael.j.lichter@nasa.gov, Not an AIAA Member.

3

Aerospace Technologist in Telecommunications, Optics and Photonics Branch (LCP), NASA GRC, daniel.e.raible@ nasa.gov, AIAA Member. 
spacecraft employing critical systems. A high speed, wide angle, nonmechanical solution is therefore desirable.

The purpose of this work is to share the development, testing, and demonstration of a breadboard prototype electro-optic (EO) scanned laser-communication link. This demonstration is a step toward realizing ultra-low Size, Weight and Power (SWaP) SmallSat/MicroSat EO non-mechanical laser beam steering modules for high bandwidth (> Gbps) free-space data links operating in the $1550 \mathrm{~nm}$ wavelength bands. The elimination of all moving parts will dramatically reduce SWaP and cost, increase component lifetime and reliability, and simplify the system design of laser communication modules. This paper describes the target mission architectures and requirements (few cubic centimeters of volume, 10's of grams of weight with milliwatts of power) and design of the beam steering module. Laboratory metrology is used to determine the component performance including horizontal and vertical resolution ( $<20$ urad) as a function of control voltage, transition time (0.1-1ms) and pointing repeatability. A test bed system demonstration, including a full laser communications link, is conducted. The capabilities of this new EO beam steerer provide an opportunity to dramatically improve space communications through increased utilization of laser technology on smaller platforms than were previously attainable.

Nomenclature

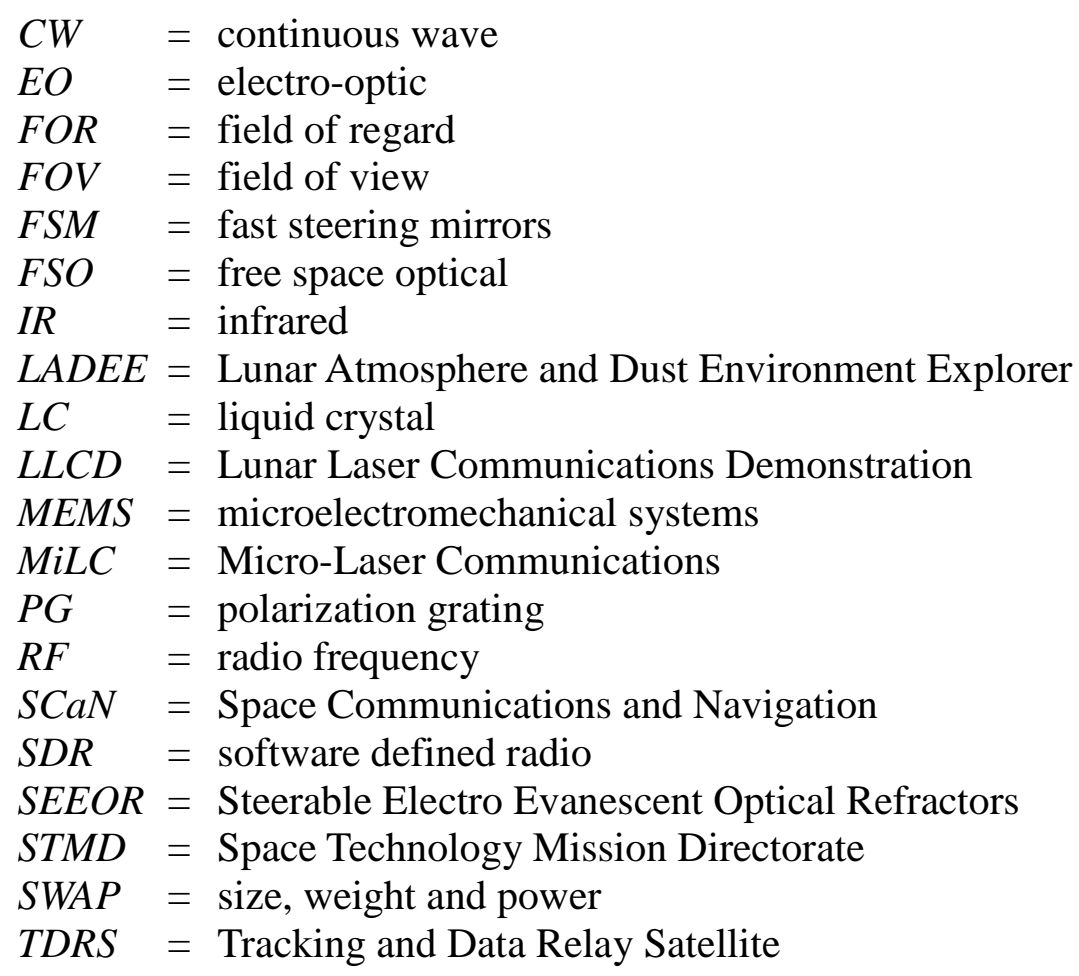




\section{Introduction and Applications}

With each advancing year, NASA directs an ever increasing amount of information gathering missions into space. As scientific instrumentation becomes more sophisticated, the amount of generated data to send to Earth is rapidly rising. Imaging and remote sensing capabilities have far outpaced our ability to transmit their data back to Earth, and this is especially true from deep space.

To accommodate this need, NASA has been migrating to higher-bandwidth radio frequencies (RF) spectrum utilization, along with increasing the size of its antennas and power of its radio transmitters. Unfortunately there exists finite limitations to the federal government's spectrum allocations, spacecraft antenna sizes are constrained by launch vehicle payload fairing sizes and RF power amplifier performance development has reached a point of diminishing returns. Removing the science data bottleneck requires new technology development, and optical communications systems seek to address theses limitations of RF communications.

Free space optical (FSO) communications within the context of NASA is a form of long distance wireless transmission of data for telecommunications across space which employ lasers instead of RF. NASA's Space Communications and Navigation (SCaN) program will utilize multiple laser transceivers throughout the network featuring optical telescopes both on the ground and in space to send and receive information between terminals. The transceivers will be able to use the infrared (IR) portion of the optical spectrum to realize communication through the implementation of optical signal modulation.

The higher carrier frequencies of optical communications offers an inherant increase in available bandwidth, meaning they can carry more mission data. The optical communications spectrum has not been regulated, so NASA does not need to consider available allocations as they do with RF. Depending on the mission application, an optical communications solution could achieve a 50\% savings in mass, $65 \%$ savings in power and up to 20x increase in data rate when compared to conventional RF systems.

The technologies to realize FSO communications have been under development for several years, and recent initiatives have aimed to migrate these systems from the laboratory to space flight demonstration. A two-way distance record for communication was set by the Mercury laser altimeter instrument aboard the MESSENGER spacecraft, and was able to communicate across a distance of 24 million $\mathrm{km}$ (15 million miles), as the craft neared Earth on a fly-by in May, 2005 (1). The previous record had been set with a one-way detection of laser light from Earth, by the Galileo probe, of 6 million km in 1992.

SCaN recently demonstrated optical communication technology at the moon with the Lunar Laser Communications Demonstration (LLCD) from October 2013 through April 2014 during the Lunar Atmosphere and Dust Environment Explorer (LADEE) mission. The LLCD demonstration consisted of a space terminal on the LADEE spacecraft and three ground terminals on Earth. Together, they demonstrated that it was possible to transfer up to $622 \mathrm{Mbps}$ of data from the Moon with a space terminal that weighs less, uses less power, and occupies less space than a comparable RF system (2). 
In conjunction with NASA's Space Technology Mission Directorate (STMD), SCaN is currently working on the Laser Communications Relay Demonstration (LCRD). LCRD will be NASA's first long-period optical communications project which will demonstrate benefits for both deep space and near Earth missions. LCRD will also validate advanced relay operation concepts which could be used for future relays, such as with Mars. The project will reutilize the ground terminals developed for LLCD, and operate at up to 1.25 Gbps (3). Finally, SCaN will introduce optical communications on an operational basis on the Next Generation Tracking and Data Relay Satellite (TDRS) in the 2022 timeframe.

For all its benefits, optical communications still faces several challenges. Unlike RF, which can be transmitted as a broad beam blanketing target areas with its signal, optical communication is sent in a relatively narrow beam pointed directly at a receiver. When broadcasting from thousands or millions of miles away, an optical communication telescope pointing must be extremely precise, as any small deviations can result in the laser missing its target entirely.

Precise pointing of the laser beam is the paramount challenge of optical communications, and is currently addressed through several systems of mechanical means including gimbals to produce large field of regard (FOR) changes, fast steering mirrors (FSM) to handle small and rapid pertubations, and vibration isolation systems to damp spacecraft oscillations from disturbing the payload. These systems enable the integration of optical communications payloads to larger spacecraft, but are restrictive toward small resource constrained platforms.

The purpose of this technology development effort is to design, test, and deliver a breadboard demonstration of an electro-optic (EO)-scanned laser-communication link which addresses the critical challenge of realizing precision laser pointing system on small spacecraft. This demonstration unit will be a step toward realizing ultra-low Size, Weight, and Power (SWaP) Micro-Laser Communications (MiLC) modules for high bandwidth (> Gbps) FSO data links operating in the 1.5 um wavelength bands. These modules will be enabled by Vescent's EO, non-mechanical laser beamsteerers. Eliminating all moving parts will dramatically reduce SWaP and cost, increase lifetime and reliability, and simplify the system design of lasercom modules.

The capabilities of MiLC are relevant across a portfolio of upcoming NASA missions. The inherent low SWaP and cost of MiLC could dramatically increase the downlink data rates from miniature satellites such as Cube-Sats. As shown in Figure 1 the ultra-low SWaP of MiLC will enable lasercom on previously unthinkable platforms. FSO lasercom on Cube-Sats or other miniature satellites could enable "on-demand" distributed networks of numerous downlink points, which would mitigate weather obscurants. This would be a new way of imagining the lasercom "network-in-the-sky". Additionally, MiLC could be used as a surface communications asset such as the 2020 rover application wherein it could provide a high bandwidth optical uplink from the surface rover to the Mars orbiter. Finally, the MiLC device could also displace mechanical fine pointing devices on larger spacecraft featuring increased aperture sizes. 


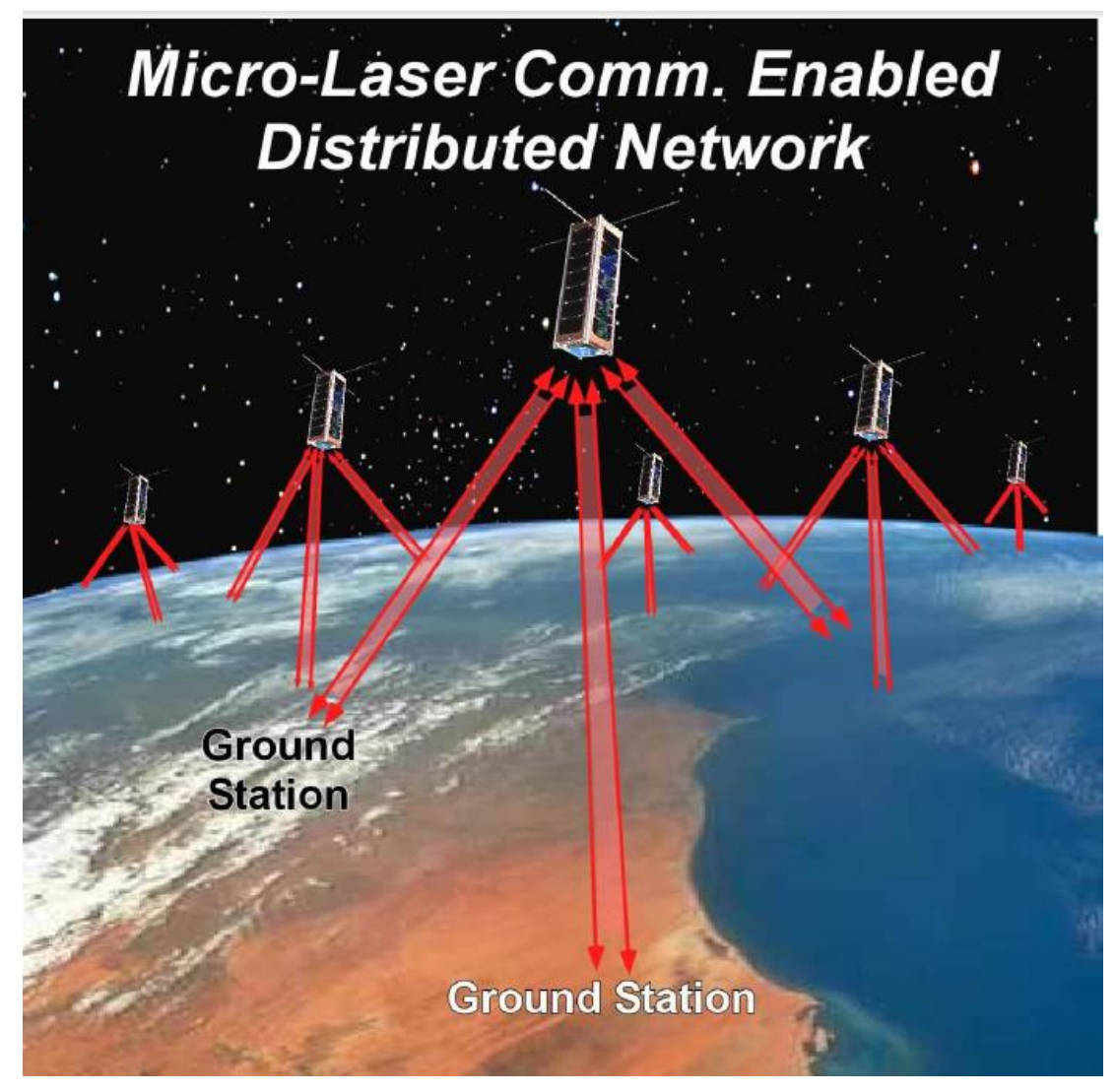

Figure 1: An example application of Micro-Laser Communications (MiLC) modules. Extremely low SWaP and cost will enable lasercom on previously unthinkable platforms.

\section{Requirements and Component Development}

EO scanners which enable continuous coverage over very wide-angles, while providing high-speed controlled sweeps over thousands of spots, all in a compact and simple package have been a long standing dream of the optics community. Past attempts have yielded wide-angle, discrete-step birefringent prisms, (4-6) but these are bulky, expensive, and slow. The design presented here replaces the prisms with a miniaturized high-speed polarization grating (PG) for the course steering stage (see upper left of Figure 2). While PGs have been studied since the 1980s, their compelling behavior was merely theoretical until recently. In 2006, Escuti et al demonstrated defect-free, 100\% efficient PGs both as switchable and polymer diffractive elements. $(7,8)$ Since these devices are not traditional gratings they exhibit very large acceptance angles while maintaining near $100 \%$ diffraction efficiency into a single order. This counterintuitive property is critical to the design. 

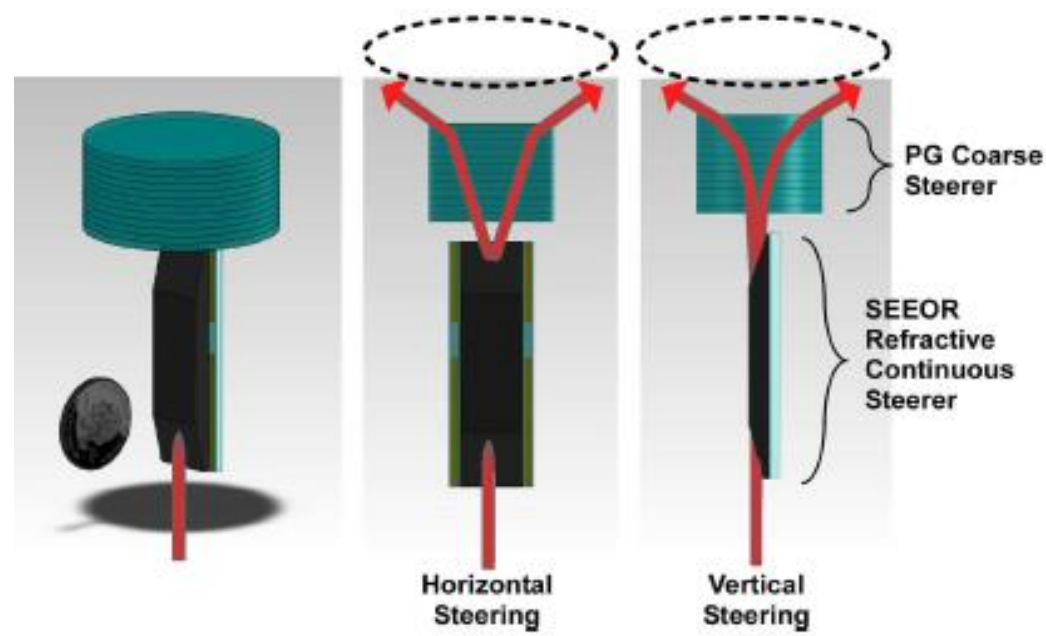

Figure 2: Illustration of PG coarse steerers combined with continuous converage SEEOR devices built at Vescent.

To fill in the gaps between the discrete angles, a continuous coverage steerer is needed. Past approaches going back decades have utilized tunable diffraction gratings, such as liquid crystal (LC) optical phased arrays, (9-11) microelectromechanical systems (MEMS) arrays, (12, 13) electro-wetting arrays, (14) and acousto-optics. Despite significant advances, (15) inherent limitations remain such as excessive dispersion due to diffraction, excessively long search time of up to several minutes, and poor coverage leading to blind spots in the field of view (FOV). (16-21) These issues are circumvented by using Vescent's Steerable Electro Evanescent Optical Refractors (SEEORs). These are enabled by the giant electro-optic effect provided by Vescent's LC-clad optical waveguides, thereby making possible unprecedented refractive EO scanners. In the enabling LC-clad architecture, the evanescent field of a waveguide mode interacts with the surface-layer LC molecules enabling electro-optic tuning of the refractive index that is five orders of magnitude larger than typical electro-optic materials like lithium niobate. This provides voltage control over optical phase on the order of millimeters.

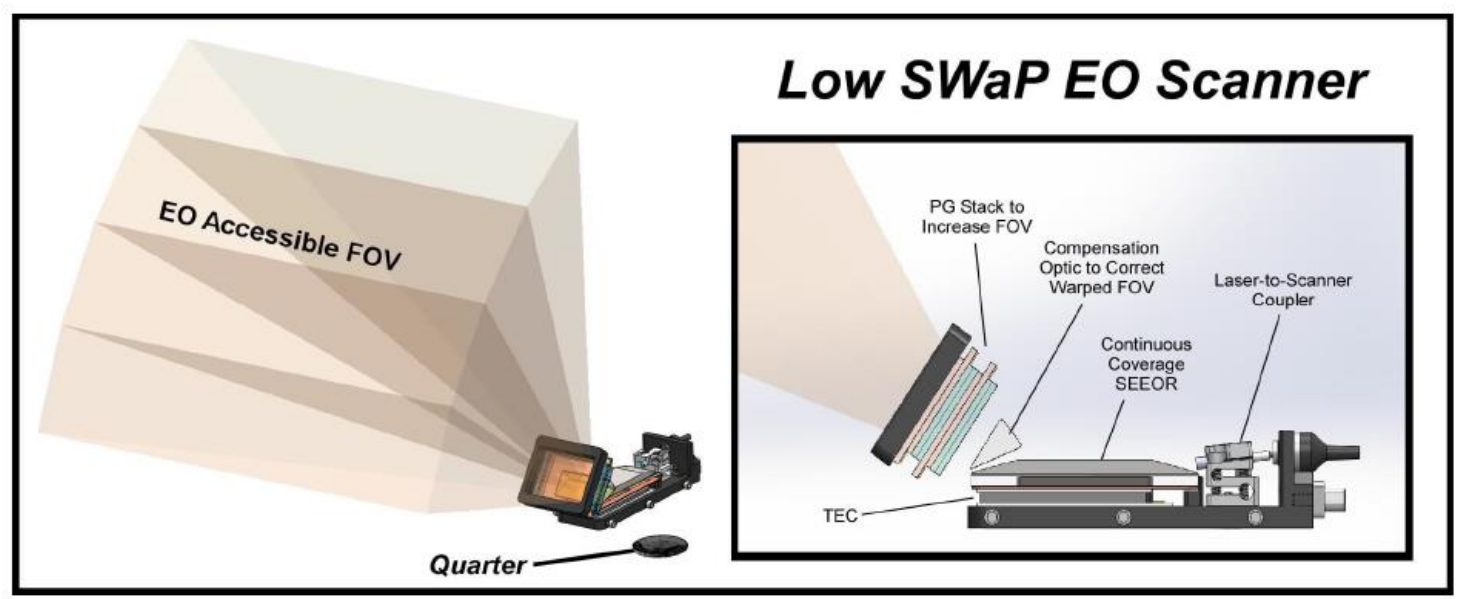

Figure 3: Co-packaged SEEOR and PG stack. 
By combining SEEOR technology with PGs a purely EO wide angle steerer has been created. In a laboratory two PG stacks were used to increase the total FOV of the refractive scanners by a factor of four, demonstrating that bigger scan angles and/or bigger beam sizes are feasible by adding more PGs. The out-of-plane scan angle is increased by choice of voltage to the PG/LC stack. The total FOV for the scanner constructed for this demonstration is approximately $18^{\circ} \times 4^{\circ}$, and a larger FOV may be easily accomplished through additional stages of polarization gratings.

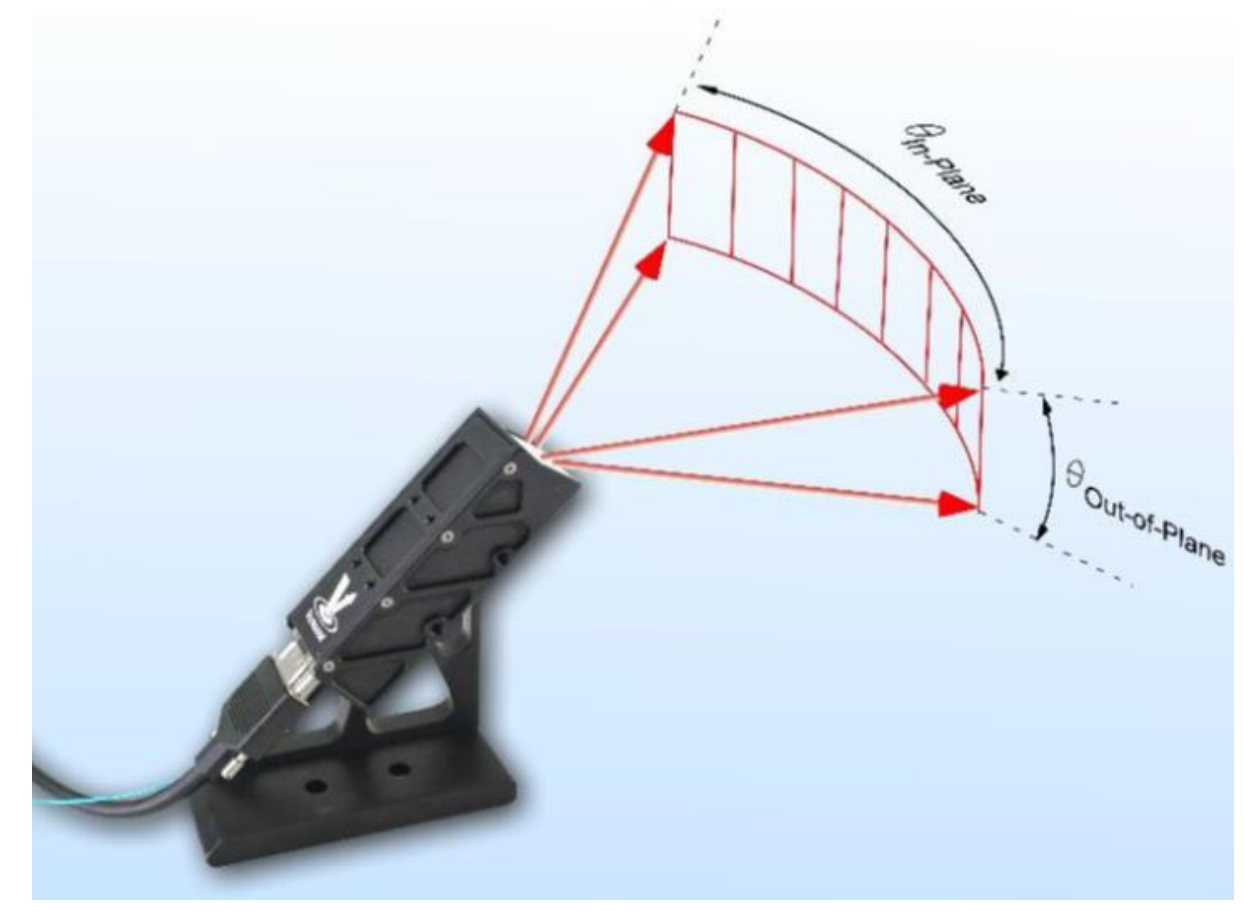

Figure 4: Pictures of the EO scanner designed and built for the demonstration.

The resulting scanner is combined with optical communications technologies to construct Micro-Laser Comm modules. MiLC modules provide a FSO capability that offers the following unique advantages:

1- Zero physical disturbance from the pointing system enables higher system pointing stability and can enable simultaneous data collections and communications.

2- Multiple downlink points: KHz bandwidth over continuous coverage wide angles provides the ability to rapidly target alternate ground receivers as an effective mitigation of the most significant FSO objection that is weather-induced blockage. By enabling the system to "instantly" switch between many ground receivers that are hundreds or thousands of $\mathrm{km}$ separated, the system will nearly always have access for data downlink.

3- Jitter compensation can be provided by the high accuracy, KHz laser beamsteerer. By closing the loop around onboard accelerometers, the system will be capable of stabilizing the downlink beam in the presence of other on-board mechanical disturbances. 
The resulting beam steering device features an ultra-low SWaP at: smaller than $7 \mathrm{x} 5 \mathrm{x}$ $5 \mathrm{~cm}$ packaging, weigh less than 10 grams and $<50$ milliWatts electrical power consumption. These features lend the MiLC device to infusion for resource constrained platforms given their inherent constraints, and the next section will describe the performance achievable from the device.

\section{Performance and Characterization}

The prototype device was characterized at the NASA John H. Glenn Research Center within the optical communications laboratory. For the first series of tests, the MiLC was mounted to a precision robotic mount capable of sub-arcsecond resolution. In this configuration the mount serves as the control and the MiLC is the variable under test. An optical quad detector was placed a distance away from the MiLC aperture to serve as a scoring system. One challenge in this configuration was matching the center of rotation of the device under test to that of the robotic actuation, so much care was taken in making these fine adjustments at the beginning of the trials.

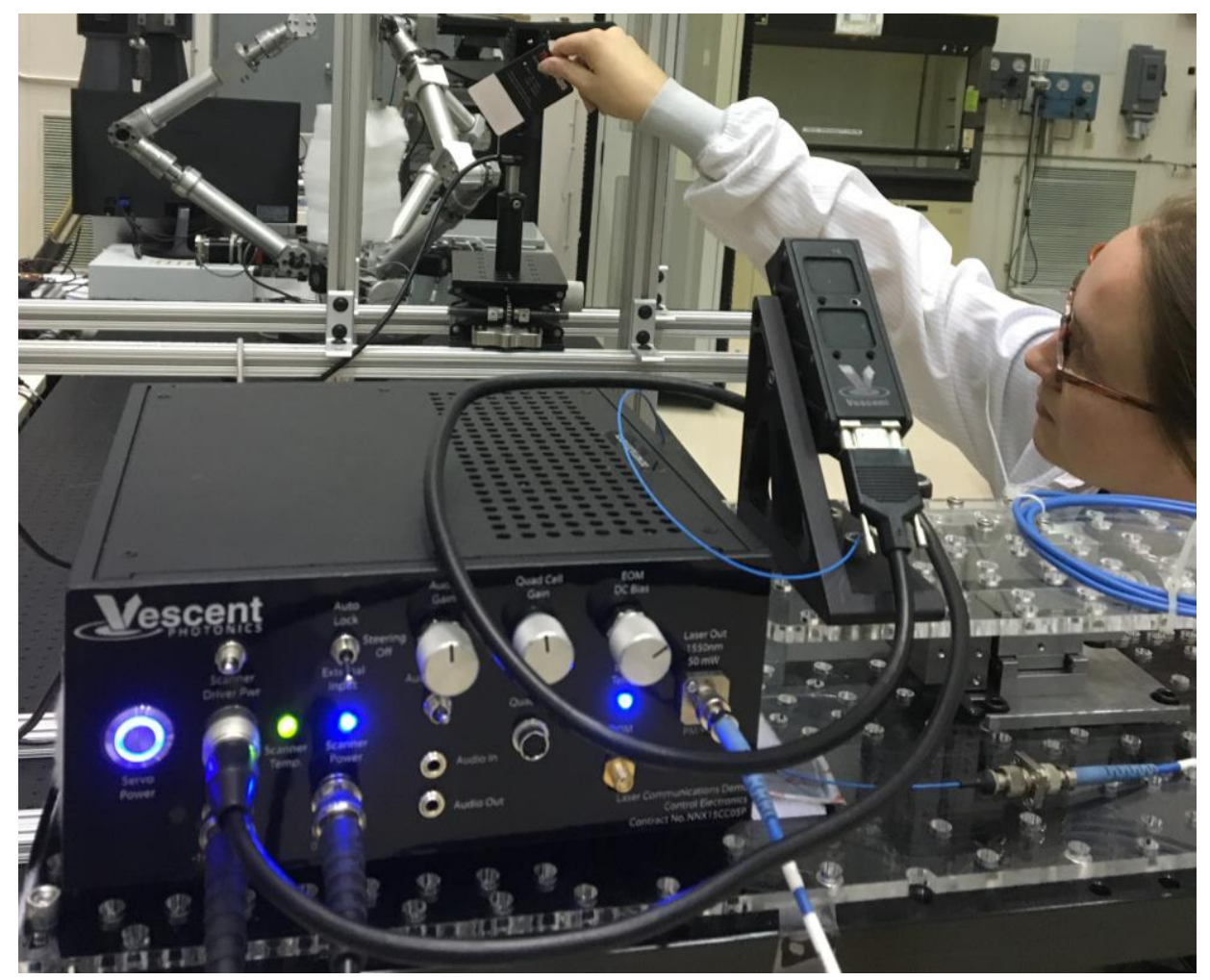

Figure 5: Calibrating the alignment of the MiLC with the metrology equipment.

The first test conducted was an independent control voltage sweep across the horizontal and vertical axes to characterize the response of the device out to its performance extents. Each control voltage was incremented from -10 to $10 \mathrm{VDC}$, while the robotic actuator was recentered to maintain boresight alignment with the quad-cell. The resulting control curves may be seen in Figures 6 and 7. Of particular note is the smooth, continuous nature of the angular system response, along with the extents agreeing favorably with the original device 
design parameters $\left(18^{\circ}\right.$ horizontal and $4^{\circ}$ vertical). An eventual flight controller could utilize these curves as look-up tables in an open-loop pointing configuration, or also as a closed-loop model based approach with augmented feedback from an on-board sensor.

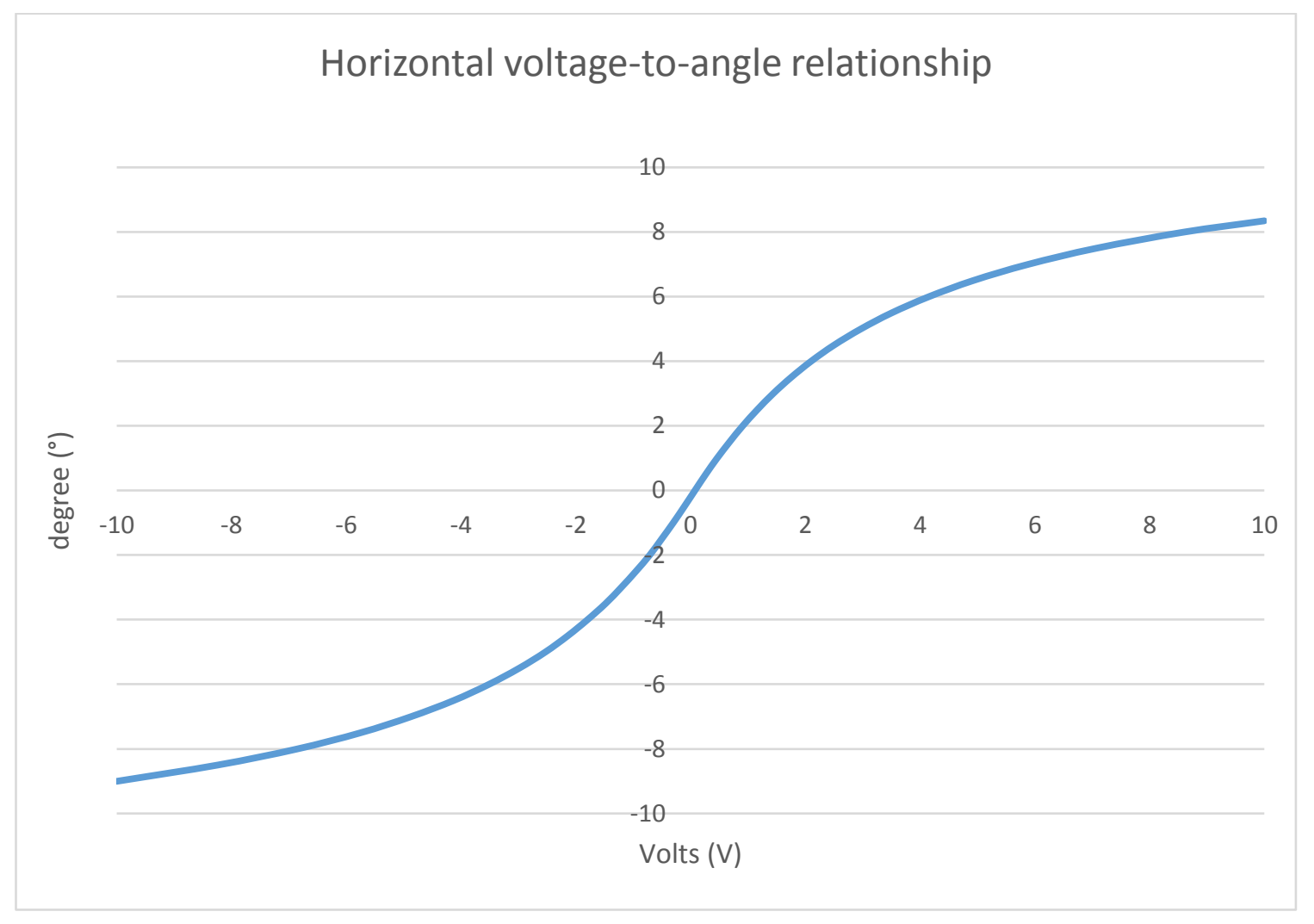

Figure 6: Scan angle versus voltage applied to the "Horizontal" input of the controller for In-Plane Steering. 


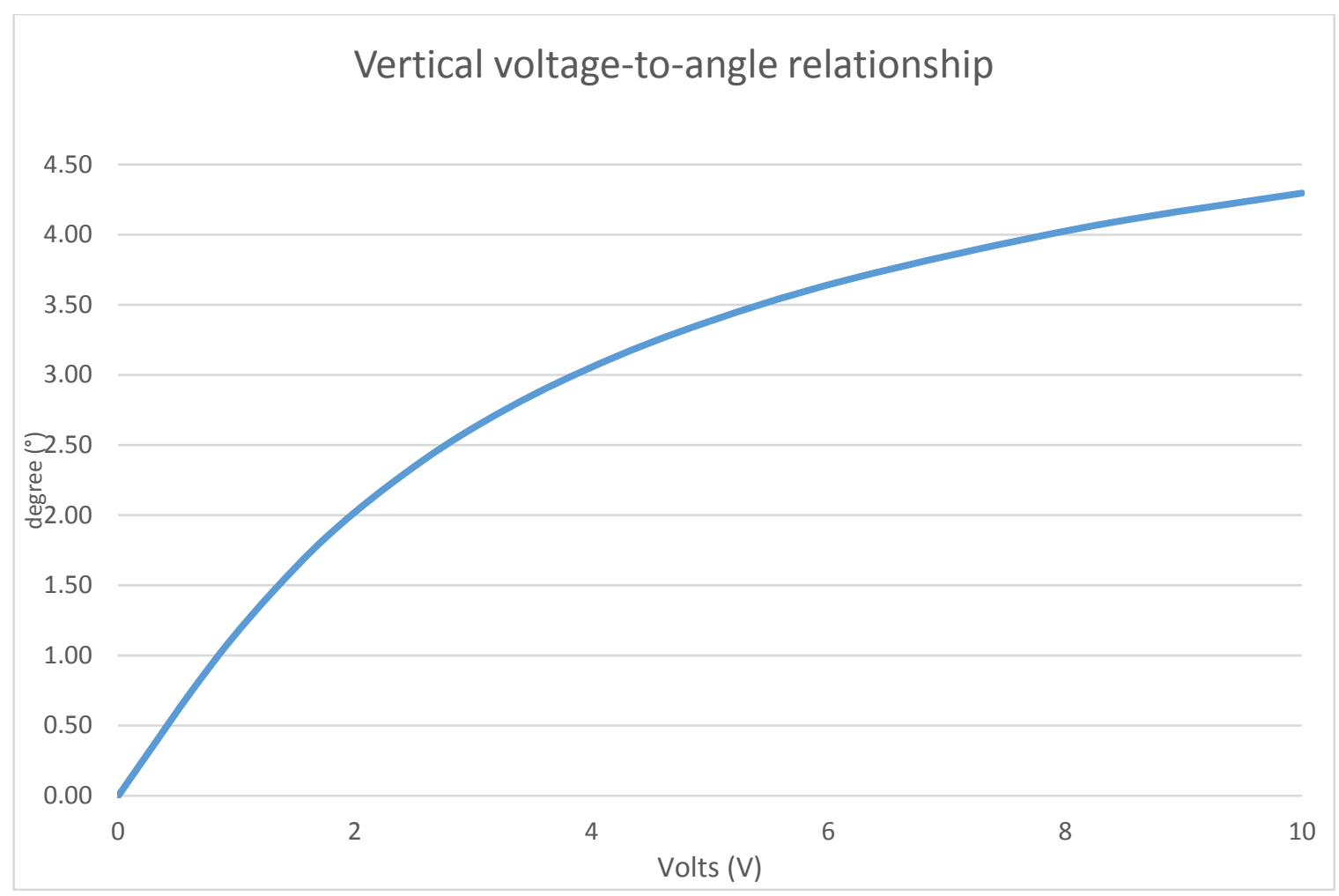

Figure 7: Scan angle versus voltage applied to the "Vertical" input of the controller for Out-Of-Plane Steering.

The next series of tests examined the angular control resolution of the MiLC device. The small indexing was made at the very limits of the metrology equipment available in the laboratory, which for this setup resulted in 0.5 arcseconds (also 0.139 millidegrees or 2.43 microradians). In the vertical direction the MiLC did in fact perform right to these measurement limits, exhibiting 0.5 arcsecond capability (and perhaps beyond our measurement ability). In the horizontal direction the MiLC was controllable down to 4 arcsecond increments (also 1.11 millidegrees or 19.40 microradians).

The next investigation describes the approach to obtain an understanding of how well the EO system repeats a commanded laser position (angle). The data taken (as described earlier) relates the voltage control to the Vescent MiLC (both horizontal and vertical dc values) to the resulting angle of the laser from the EO head. This is represented by:

$$
a=f(c v)
$$

This relationship is shown in the plots of the data taken in figures 6 and 7 (this is the data taken using the robotic mount). With this data, one can know the angle out of the Vescent MiLC with any control voltage.

For the repeatability test, a Matlab script was written to control an InfiniiVision 6000 scope's waveform generators 1 and 2, and acquire and log the voltage values read from the quad sensor (Thorlabs PDQ30C); this was done using the Matlab Instrument Control 
Library. The test run consisted of controlling the Vescent system to point repeatedly at a predefined arbitrary point on a quad sensor, after just having pointed at a random point. The number of times this was done was $\mathrm{N}=500$, and the rand function was used to generate the random point. The quad sensor was read after each control movement.

From equation (1) above, the value of the control voltage can be related to the value of degrees, and after this connection is made for the repeatability test, the data is shown in figure 8 below. The plot has in it only the fixed point value points on the quad, and has left out the random points. Also on the plot are the computed average and standard deviation in volts, and the corresponding standard deviation in urads and arcseconds. As background, the data for this repeatability was done at a different time, and for calibration, the voltage from the quad was measured while scanning through the control values of interest, and a curve fit was generated relating the two, giving $a=f($ quad_v $)$.

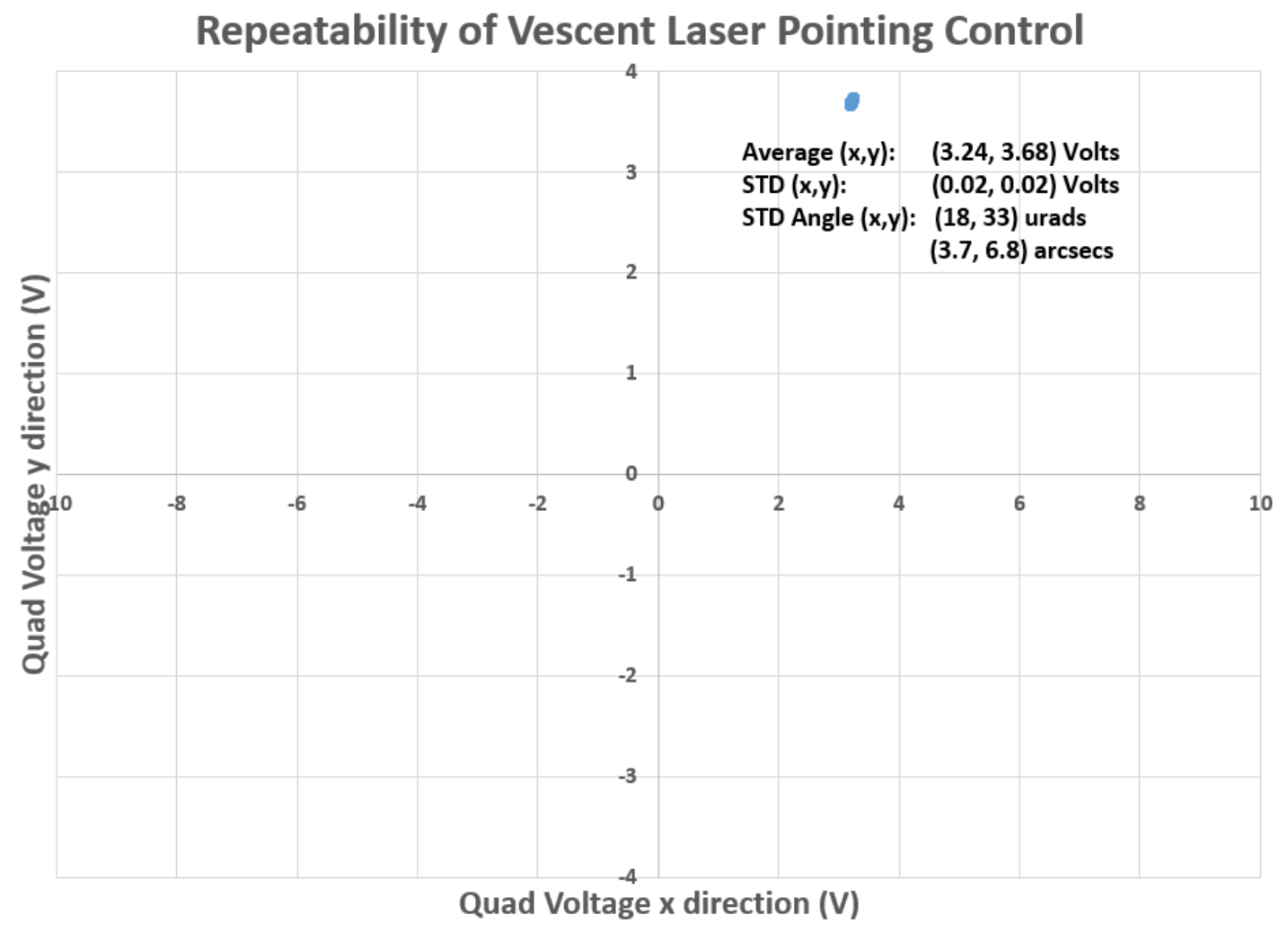

Figure 8: Data taken using the Vescent EO System to understand the repeatability of the control system.

Note that figure 8 represents just a small fraction of the Vescent system's total FOV. This is because the quad sensor only measures $3 \mathrm{~mm}$ by $3 \mathrm{~mm}$ and was a stationary object for data acquisition.

After determining the empirical FOV limits, characteristic curve, incremental step resolution, and repeatability, the next step was to measure the system response time. The MiLC was commanded to one of the full extents in the horizontal direction, and then given a step command to scan to the opposite extent. This event was measured to take $50 \mathrm{~ms}$ to 
cover the $18^{\circ} \mathrm{FOV}$, resulting in a scan rate of 360 degree/s (Figure 9). For a system identification perspective, a step input to the MiLC yielded a time constant $(\tau)$ of $1.67 \mathrm{~ms}$, yielding an effective first order transfer function of:

$$
\mathrm{H}(\mathrm{s})=1 /(0.00167 \mathrm{~s}+1)
$$

As built, the system exhibits a bandwidth of $95.24 \mathrm{~Hz}$, which is certainly adequate for many vibration stabilization tasks. For a particular bandwidth implementation the device could be redesigned to further optimize for transition time rather than FOV.

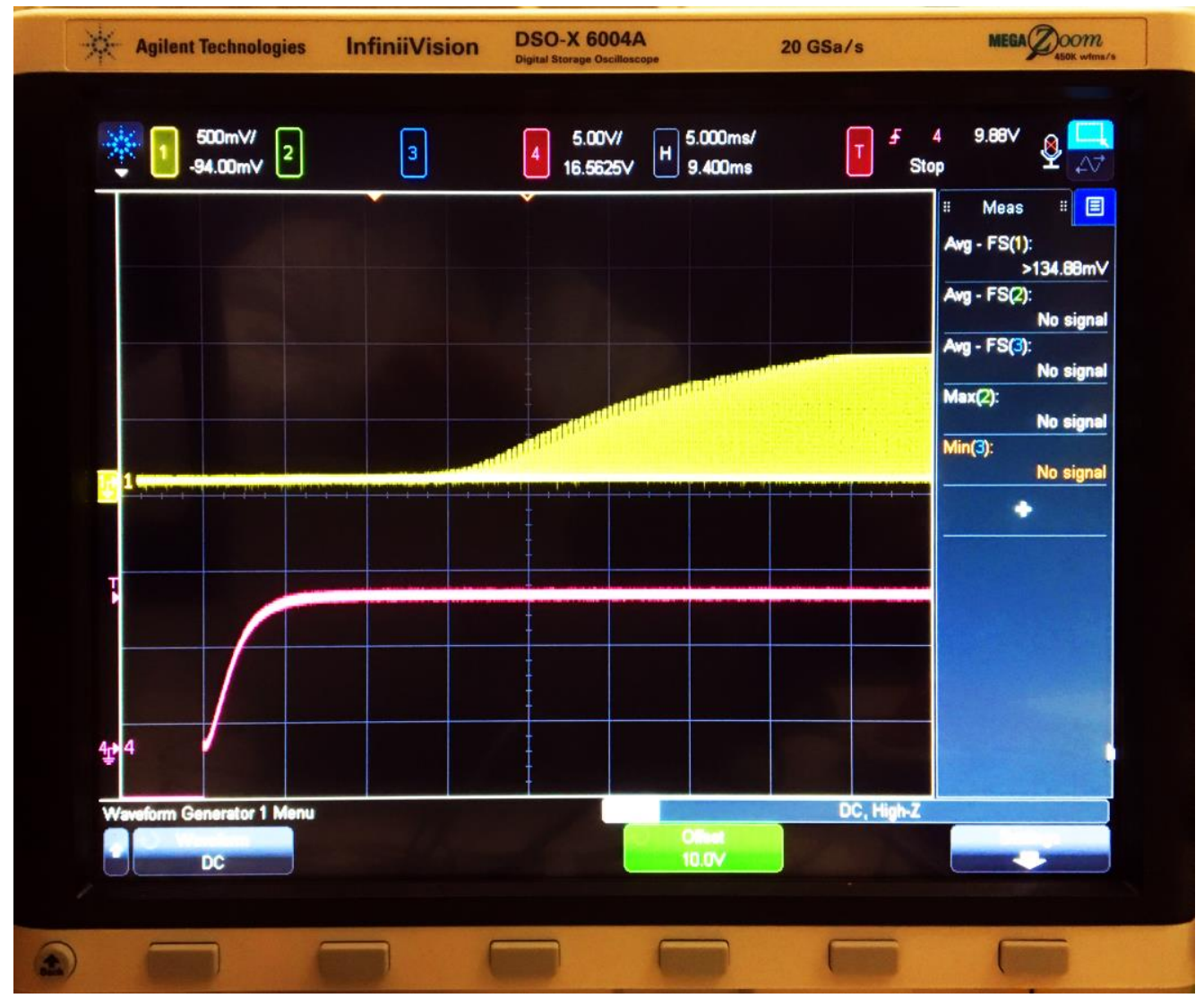

Figure 9: MiLC system response (yellow trace) to a full angle deflection control signal (purple trace).

Finally, it is worth pointing out that the SEEOR can handle higher CW optical powers than the device demonstrated here, and has excellent output beam quality. Vescent conducted a test of the device with a 5 Watt cw laser input for a full 24 hours with no signs of degradation utilizing a $1 \mathrm{~mm}$ beam diameter. It follws that increasing the beam diameter will spread the power out and enable even larger CW optical powers, but for the foreseeable future 5 watts is more than sufficient for proximity architectures in space laser communications. Second, the beam quality exiting the SEEOR is comparable to a beam exiting a single mode fiber, and has been measured to have an $\mathrm{M}^{2}$ of 1.02 . 


\section{Future Work}

The electronics package and optical head together provide everything necessary for setting up and running an EO, i.e. non-mechanically, stabilized optical data transmission. The system is capable of transmitting both a high-speed data signal and an audio signal $(20-20 \mathrm{kHz})$, which are modulated onto the laser before being output from the electronics package. The modulated laser beam is then guided by the EO beam-steering device to track and lock to a detector, and maintain a data link.

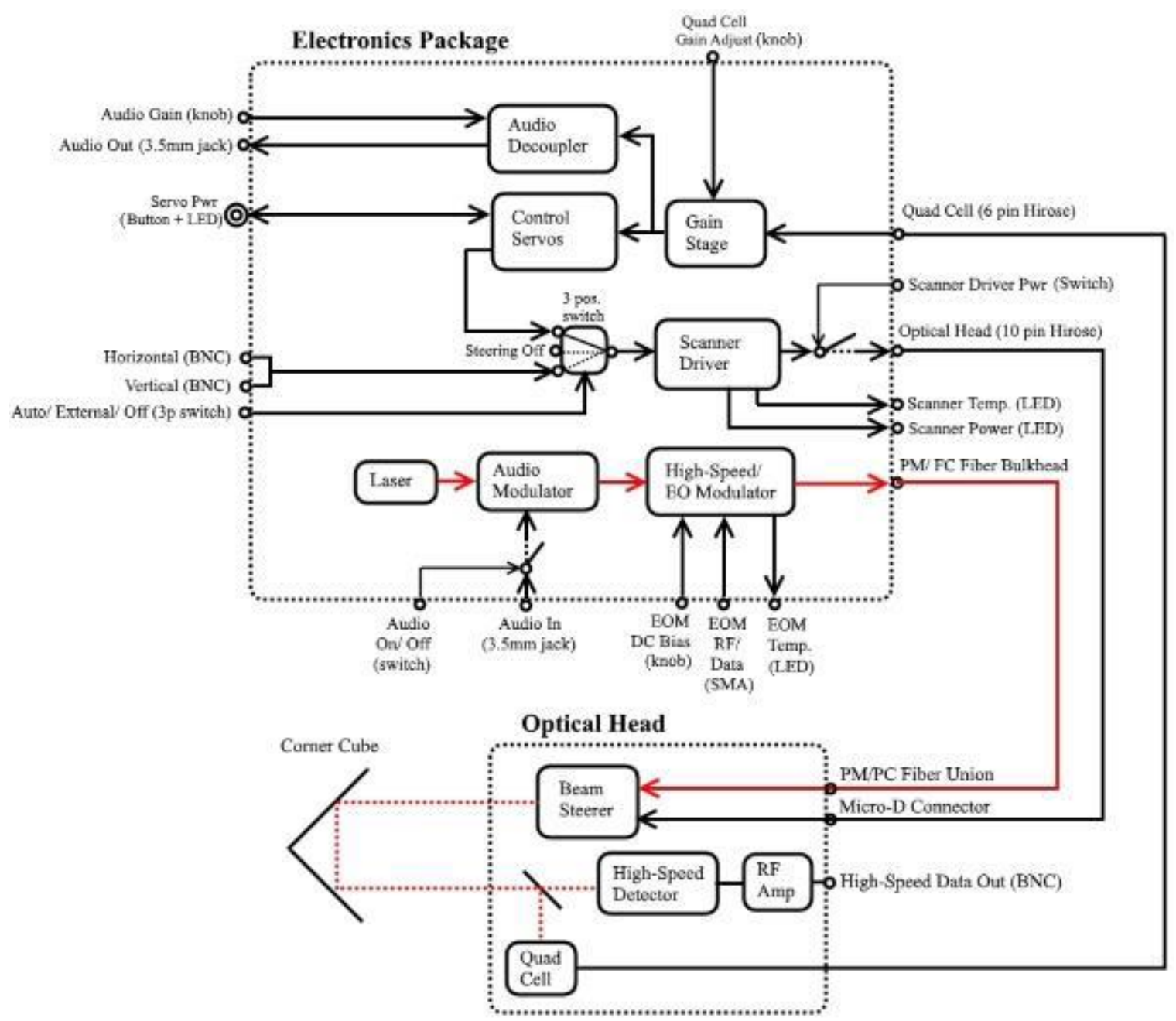

Figure 10: Functional Block Diagram of EO Scanned Laser Communication

The electronics package contains the source laser, the locking servos, the scanner control driver, and the modulation circuitry. In auto-lock mode, the scanner will sweep the laser beam across its FOV until laser light hits the detector system. At this time the locking servos are engaged and the scanner will adjust the beam angle to keep it aligned to the detectors.

Together with the scanner, the resultant package is easily integrable with additional subsystems, so the natural progression is to merge this with other prototype technologies 
to conduct complete end-to-end systems tests. Concurrently under development are low wall-plug efficient lasers and optical software defined radios (SDR) targeted toward small satellite and rover applictions. These subsystems are planned to be combined with the MiLC architecture above to demonstrate complete and feasible compact and lightweight optical communication systems through field testing.

\section{Conclusion}

The era of operable FSO communications systems is upon us, so much like the decades of microwave-based RF capability development and refinement following WWII. We are now entering a similar period which will see an explosion of new enabling technologies to expand the realm of optical communications. Precision, compact, and low power laser pointing solutions need to be at the forefront of these developments in order to spur the adoption of FSO systems across a range of host platforms.

Here we have demonstrated the feasibility of adapting novel electro-evanescent refractive beamsteerers to adress lasercom needs, and especially so for small resource constrained platforms. The fundamental performance of a prototype example of the technology has been quantified in terms of controllability, resolution, repeatibility and transition time. In on-going efforts we will further integrate this technology with other emergent FSO technologies optimized to meet the requirements of NASA lasercom missions including near and deep space cubesat optical links, planetary lander/rover optical proximity links, and potential beam stabilization of large FSO systems.

\section{Acknowledgments}

The authors would like to thank the NASA Space Communications and Navigation (SCaN) program, and in particular Dr. Don Cornwell for supporting this research. In addition, we would like to thank the NASA Small Business Innovation Research (SBIR) program for contract NNX15CC05P, and also Ms. Kristina Collins for her attention to detail and diligent laboratory contributions.

\section{References}

[1] "Space probe breaks laser record: A spacecraft has sent a laser signal to Earth from 24 million km (15 million miles) away in interplanetary space". BBC News. January 6, 2006. Retrieved June 28, 2011.

[2] B. S. Robinson ; D. M. Boroson ; D. A. Burianek and D. V. Murphy, "Overview of the lunar laser communications demonstration", Proc. SPIE 7923, Free-Space Laser Communication Technologies XXIII, 792302 (February 21, 2011); doi:10.1117/12.878313.

[3] Bernard L. Edwards; Dave Israel; Keith Wilson; John Moores and Andrew Fletcher, "Overview of the Laser Communications Relay Demonstration Project," SpaceOps 2012 Conference, http://dx.doi.org/10.2514/6.20121261897.

[4] 2. S. A. Kahn, and N. A. Riza, "Demonstration of 3-dimensional wide angle laser beam scanner using liquid crystals," Optics Express 12, 868-882 (2004).

[5] H. Meyer, D. Riekmann, K. P. Schmidt, U. J. Schmidt, M. Rahlff, E. Schrbder, and W. Thrust, "Design and performance of a 20-stage digital light beam deflector," Applied Optics 11, 1932-1936 (1972).

[6] U. Schmidt, and W. Hust, "Optical deflection system including an alternating sequence of birefringent prisms and polarizers," U.S. Patent 3,572,895, (1986).

[7] J. Kim, C. Oh, M. J. Escuti, L. Hosting, and S. A. Serati, "Wide-angle, nonmechanical beam steering using thin liquid crystal polarization gratings," in Advanced Wavefront Control: Methods, Devices, and Applications VI, (SPIE, 2008), pp. 709302-709301.

[8] S. R. Nersisyan, N. V. Tabiryan, D. M. Steeves, and B. R. Kimball, "The principles of laser beam control with polarization gratings introduced as diffractive waveplates," in Proc of SPIE I. C. Khoo, ed. (2010), pp. 77750U77751. 
[9] J. Borel, J.-C. Deutch, G. Labrunie, and J. Robert, "Liquid Crystal Diffraction Grating," U. S. P. Office, ed. (Commissariat A L'Energie Atomique, 1974).

[10] J. P. Huignard, M. Malard, and G. d. Corlieu, "Static Deflector Device for An Infrared Beam," U. S. P. a. T. Office, ed. (Thomson-CSF, USA, 1987).

[11] P. McManamon, P. J. Bos, M. J. Escuti, J. Heikenfeld, S. A. Serati, H. Xie, and E. A. Watson, "A Review of Phased Array Steering for Narrow-Band Electrooptical Systems," Proceedings of the IEEE 97, 1078-1096 (2009).

[12] R. Ryf, H. R. Stuard, and C. R. Giles, "MEMS tip/tilt \& piston mirror arrays as diffractive optical elements," Proceeding of SPIE, Bellingham, WA 5894, 58940C-58941-58911 (2005).

[13] K. Krishnamoorthy, K. Li, D. Yu, D. Lee, J. P. Heritage, and O. Solgaard, "Dual mode micromirrors for optical phased array applications," Sensors and Actuators A A97-98, (2002).

[14] N. R. Smith, D. C. Abeysinghe, J. W. Haus, and J. Heikenfeld, "Agile wide-angle beam steering with electrowetting microprisms," Optics Express 14, 6557-6563 (2006).

[15] http://www.bnonlinear.com/products/1x12288Linear/1x12288Linear.html.

[16] Y. Chiu, R. S. Burton, D. D. Stancil, and T. E. Schlesinger, "Design and Simulation of Waveguide Electrooptic Beam Deflectors," Journal of Lightwave Technology 13, 2049 (1995).

[17] Y. Chiu, J. Zou, D. D. Stancil, and T. E. Schlesinger, "Shape-Optimized electrooptic beam scanners: Analysis, design, and simulation," Journal of Lightwave Technology 17, 108 (1999).

[18] J.-h. Kim, L. Sun, C.-h. Jang, C.-C. Choi, and R. T. Chen, "Polymer-based thermo-optic waveguide beam deflector with novel dual folded-thin-strip heating electrodes," Optical Engineering 42, 620-624 (2003).

[19] D. A. Scrymgeour, Y. Barad, V. Gopalan, K. T. Gahagan, Q. Jia, T. E. Mitchell, and J. M. Robinson, "Largeangle electro-optic laser scanner on LiTaO3 fabricated by in situ monitoring of ferroelectric-domain micropatterning," Applied Optics 40, 6236 (2001).

[20] J. E. Stockley, S. A. Serati, G. D. Sharp, P. Wang, K. F. Walsh, and K. M. Johnson, "Broadband Beam Steering," in SPIE Proceedings, (1997).

[21] K. Nakamura, J. Miyazu, Y. Sasaki, T. Imai, M. Sasaura, and K. Fujiura, "Space-charge-controlled electro-optic effect: Optical beam deflection by electro-optic effect and space-charge-controlled electrical conduction," Journal of Applied Physics 104, 013105-013101 (2008). 